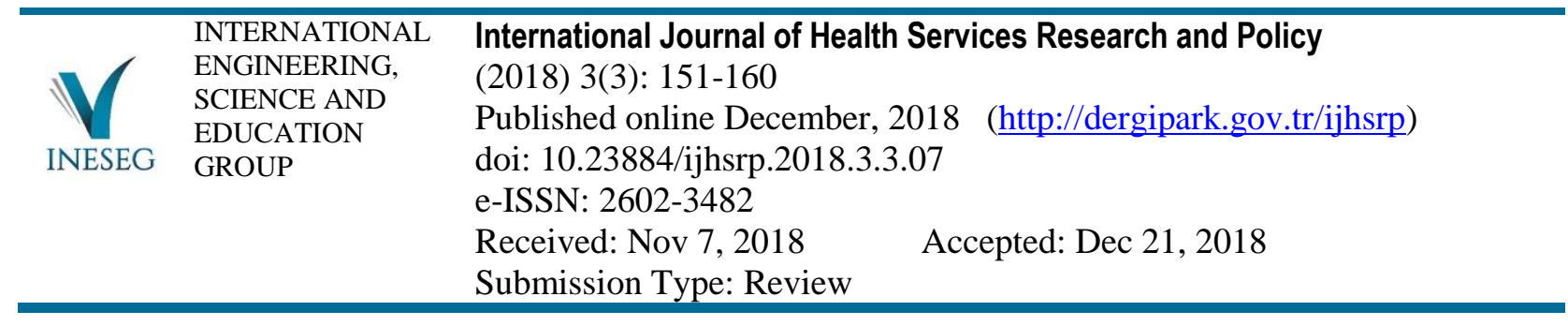

\title{
CHANGABLE ENVIRONMENTAL RISK FACTORS IN AUTISM: VITAMIN D AND FOLIC ACID
}

\section{Nalan Hakime NOGAY ${ }^{1}$}

${ }^{1}$ Erciyes University Faculty of Health Sciences, Department of Nutrition and Dietetics, Kayseri

* Corresponding author; nalanhakime@gmail.com

\begin{abstract}
Autism Spectrum Disorder (ASD) is a severe neuro-developmental disorder which generally starts during infancy as well as mostly in the first 3 years of life. ASD is characterized by social, behavioral, and communication symptoms. Genetics and certain environmental factors may play a role in the pathogenesis of autism, although the etiology of the disease is unknown. Maternal nutrition is important for development of the fetal brain. The mother's nutritional condition before and during pregnancy may affect the risk of ASD incidence. Vitamin D deficiency is taken into consideration as a possible risk factor for development of ASD. Compared to healthy peers, children with ASD were indicated to have lower levels of vitamin D. However, it is not clear yet if this condition was influenced by the fact that whether patients with ASD are born with lower levels of vitamin D or they are exposed to sunlight lesser. Also folic acid supplementation during pregnancy and blood folate levels in children with autism are environmental factors contributing to autism incidence through dietary intake. The aim of this study is to explain the relationship between vitamin D, folic acid and ASD.
\end{abstract}

Key Words: Autism, Folic acid, Vitamin D

\section{Introduction}

Autism Spectrum Disorder (ASD) is a severe neuro-developmental disorder which generally starts during infancy as well as mostly in the first 3 years of life [1]. ASD is characterized by social, behavioral, and communication symptoms [2]. It has been recently shown that prevalence of ASD has increased dramatically [3].

Genetics and certain environmental factors may play a role in the pathogenesis of autism, although the etiology of the disease is unknown. Exposure to prenatal teratogens, alcohol, and infections are factors that trigger autism incidence [4].

Maternal nutrition is important for development of the fetal brain. Insufficient maternal nutrition is related to an increase in negative neurodevelopmental results such as neural tube defect, autism spectrum disorder (ASD), and schizophrenia. The fetal brain's structural and functional development is affected by nutrient balance and need. Therefore, the mother's nutritional condition before and during pregnancy may affect the risk of ASD incidence [5]. Along with neurological disorders, metabolic disorders such as phenylketonuria, histidinemia, mitochondrial dysfunction, chronic constipation, stomachache, 
malabsorption, gluten intolerance, and allergies are observed. Besides gastrointestinal symptoms, children with autism have nutrition problems such as preferring specific tastes, disgusting certain textures, preferring soft and sweet foods. Thus, nutritional deficiencies play a role in autism [6].

Photolabile and photosynthetic vitamins lead to a higher autism risk by altering genetic and epigenetic interactions. During pregnancy, folic acid and Vitamin D, which are photolabile vitamins, are quite important [7].

Folic acid supplementation during pregnancy and blood folate levels in children with autism are environmental factors contributing to autism incidence through dietary intake. Plasma phosphate concentration is an important determiner of homocysteine levels and a negative correlation exists between them. Serum homocysteine levels are higher in patients with ASD than in their healthy peers. In addition, vitamin $\mathrm{B}_{12}$ is essential for metabolism of homocysteine [4].

Vitamin D deficiency is taken into consideration as a possible risk factor for development of ASD [8]. Compared to healthy peers, children with ASD were indicated to have lower levels of vitamin D. However, it is not clear yet if this condition was influenced by the fact that whether patients with ASD are born with lower levels of vitamin D or they are exposed to sunlight lesser [2]. Vitamin D deficiency is one of the most critical risk factors of ASD due to several reasons. Firstly, epidemiologic information indicates that vitamin D deficiency is a risk factor for ASD. Secondly, decreased levels of serum vitamin D are associated with alexithymia seen in ASD. Thirdly, vitamin D takes part in DNA repair. Vitamin D can reduce severity of ASD by showing an anti-inflammatory and autoimmune effect and also increasing the number of T-regulatory cells [9]. The aim of this study is to explain the relationship between vitamin $\mathrm{D}$, folic acid and ASD.

\section{VITAMIN D}

\subsection{Vitamin D and risk of ASD}

Vitamin D is a steroid hormone that can be taken by diet. However, vitamin D synthesized from sunlight in the skin is the essential resource of vitamin D. Production of vitamin D by the skin is extraordinarily strong and fast. For example, light-skinned people produce vitamin D more than 20000 IU in a time shorter than $30 \mathrm{~min}$ when whole of their bodies are exposed to sun in the summer [10]. While Serum 25(OH) D concentration of $<25 \mathrm{nmol} / \mathrm{L}$ is defined as vitamin D deficiency, the concentration of $25-49.9 \mathrm{nmol} / \mathrm{L}$ is defined as vitamin $\mathrm{D}$ insufficiency [11]. The best known role of vitamin $\mathrm{D}$ is that it facilitates absorption of calcium and phosphate in the intestine [12]. It also plays role in neurodevelopment, immunological modulation, antioxidant mechanisms, and gene regulation. Positive effects of vitamin D on the human body, particularly on the brain, are known well. Vitamin D deficiency poses a major health problem for numerous societies [13]. Factors such as exposure to sunlight, skin pigmentation, season of delivery, and location of residence affect level of vitamin D [1]. It was reported that risk of developing ASD in further life was higher in children whose mother became pregnant in winter months compared to those whose mother became pregnant in summer months [11]. In a related study, risk of ASD stated to increase by $6 \%$ in children whose mother became pregnant in winter season in California between 1990 and 2002 compared to summer season [14]. Another issue focused by scientists is the relationship between season of delivery and ASD risk. Despite the fact that different results were obtained from previous studies, the season in which child is born is considered to be likely associated with ASD risk [11]. A study on vitamin D level of mother and risk of ASD for her child reveled that lower $25(\mathrm{OH})$ D levels in pregnancy were associated with occurrence of more mental 
and psychomotor problems in child to be born [15]. On the other hand, in another study, no relationship was found between maternal $25(\mathrm{OH}) \mathrm{D}$ concentration and psychological health and intelligence of the child at the age of 9 months and 9 years [16]. Obtaining different results from the studies is thought to be arising from differences in timing of blood samples taken during pregnancy. In addition, these studies show that different parts of infants' brains may be affected depending on timing of exposure to low level of vitamin D during pregnancy and this leads to cause different neurodevelopmental and cognitive outcomes [11].

Vitamin D levels of 13 children with ASD and 14 healthy children without any medical or psychiatric disorder, aged between 3 and 12 years, were measured and it was revealed that there was no difference between two groups and no relationship between vitamin D level and autism [8]. In an study conducted with 237 children with ASD and 243 healthy children in order to determine the effect of VDR polymorphism on serum $25(\mathrm{OH}) \mathrm{D}$ level, significant correlation was determined between VDR (vitamin D receptor) polymorphism and ASD supporting the hypothesis that $25(\mathrm{OH}) \mathrm{D}$ plays role on pathology of autism [1]. Vitamin D affects development of the brain through endocrine functions such as stressmediated agents, inflammation mediated cytokines, and regulation of extracellular calcium. Vitamin D also has direct effects on the brain. Vitamin D has an effect on the brain development via regulation of calcium and glucocorticoids in embryonic neurons, regulation of neurotrophic factors in embryonic brain, embryonic neuronal differentiation, regulation of reactive oxygen species in embryonic nonneuronal cells, and anti-inflammatory effect on developing brain [17].

\subsection{The action mechanism of vitamin $D$ in ASD}

Some possible mechanisms of therapeutic and preventative effects of vitamin D in ASD have been discussed. One of these is to make contribution to vitamin D for DNA repair. Vitamin D deficiency takes place in numerous de novo gene mutation. Reactive oxygen species are a part of normal metabolic function of somatic cells and regularly contribute to DNA damage. Therefore, human genome needs to repair itself [11]. Researchers have reported that vitamin D deficiency is associated with DNA damage and sufficient levels of vitamin D are critical for preventing DNA damage [10]. The second one is the major role of vitamin D in immune system. Evidences show occurrence of neuroglial activation and neuroinflammation of the brains of patients with ASD [2]. Recent studies indicate that children with ASD have lower levels of glutathione and glutathione peroxidase. Oxidative stress and reduced antioxidant capacity were revealed to result in chronic inflammatory response, mitochondrial dysfunction, DNA damage, and clinical characteristics of autism [11]. Vitamin D increases production of antioxidants such as thioredoxin, glutathione, and superoxide dismutase [2]. Glutathione protects nerve cells against toxins. Thirdly, in ASD, the existence of maternal antibodies in fetal brain tissue was shown. Level of these autoantibodies was thought to be directly associated with severity of autism. Vitamin D has a stimulatory effect on T regulatory cells. T regulatory cells suppress reactions by other autoimmune cells in order to prevent body to attack its own tissues [10]. Thus, vitamin D may have a role to protect fetus and decrease autoimmune conditions by stimulating $T$ regulatory cells [2]. In a study conducted about this topic, it was reported that $73.3 \%$ of children with autism had $\mathrm{T}$ regulatory cell deficiency [18].

ASD, also, is an inflammatory disease. While Vitamin D increases protective immune response, it decreases inflammation. Calcitriol regulates various immune cells such as monocytes, macrophages, and $\mathrm{T}$ and B lymphocytes. In studies on patients with ASD, proinflammatory molecules like MCP-1, 
TNF- $\alpha$, were observed to increase. Vitamin D, on the other hand, has a decreasing effect on these molecules [10].

Neurotrophins are the proteins stimulating nerve and brain cells to survive, develop, and function [1]. It was indicated that levels of several neurotrophic factors such as nerve growth factor (NGF) and brain derived neurotrophic factor (BDNF) were abnormal in some individuals with ASD [11]. Vitamin D takes part for regulation of neurotrophins. Mitochondrial disorder is seen in 1 out of every 20 people with ASD. Vitamin D is considered to recover mitochondrial damage[10].

Seizure is observed in more than $25 \%$ of patients with ASD. In a previous study, vitamin D deficiency was determined in 8 of 13 patients suffering seizures and these individuals were administered 40000 200000 IU vitamin D for once, and then they were continued to be administered 2000-2600 IU/day for 3 months. As a result of the study, seizures were detected to decrease by $40 \%$ [19].

Another effect of vitamin D is on serotonin. While active form of vitamin D decreases peripheral TPH1, it increases central TPH2; this condition explains serotonin paradox in ASD, that is peripheral serotonin is high but central serotonin is low [3]. Vitamin D regulates serotonin synthesis in the brain through tryptophan hydroxylase 2 (TPH2). Therefore, low vitamin D levels result in abnormal serotonin synthesis causing abnormalities in brain development. Low vitamin D levels may result in insufficient TPH2 expression during fetal and neonatal development and lead to low serotonin concentration in the developing brain [20].

\subsection{The use of vitamin $D$ for treating and preventing ASD}

It has been hypothesized that gestational vitamin D deficiency contributes the cause of autism. Vitamin D supplementation during pregnancy and early childhood is also considered to decrease prevalence of autism in newborn siblings. In a related study, mothers of children with ASD were given $5000 \mathrm{IU} /$ day vitamin D during pregnancy and their newborns were given $1000 \mathrm{IU} /$ day until the age of 3 years, and only 1 of 19 children followed up was diagnosed with ASD. It was concluded in the study that vitamin D administered to infants and toddlers during pregnancy and after birth could decrease incidence of autism [2].

Vitamin D supplementation is thought to decrease behavioral problems of children with ASD. In a related study, 37 of 215 children with ASD were administered with 150000 IU once a month and $400 \mathrm{IU} /$ day for 3 months and it was observed that $25(\mathrm{OH}) \mathrm{D}$ levels of all children increased and their behavioral problems recovered at the end of 3 months [13]. In another study, individuals with ASD, having averagely $31.5 \mathrm{nmol} / \mathrm{L}$ of baseline $25(\mathrm{OH}) \mathrm{D}$ level were administered with daily 1600-4000 IU $\mathrm{D}_{3}$ and the depression was recovered [21]. 106 children with ASD, possessing serum 25(OH) D concentration of $<75 \mathrm{nmol} / \mathrm{L}$, were administered $300 \mathrm{IU} / \mathrm{kg}$ vitamin D for 3 months in such a way not to exceed 5000 IU per day, and behaviors such as irritability, stereotypic behavior and hyperactivity considerably recovered [22]. In a study including 60 children with ASD and control group, aged between 4 and 10 years, $78.3 \%$ of children with ASD and $33.3 \%$ of control group were observed to have sleep disorders. In the same study, it was observed that group with serum 25(OH) D concentrations of 20-29 $\mathrm{nmol} / \mathrm{L}$ was administered daily $5000 \mathrm{IU}\left(\mathrm{D}_{2}\right)$, the group with $<20 \mathrm{nmol} / \mathrm{L}$ received weekly 50000 $\mathrm{IU}\left(\mathrm{D}_{2}\right)$ and vitamin D supplementation had a positive effect on total duration of sleep [23].

Vitamin D is a potential preventative agent for ASD due to its effects such as anti-inflammatory effects, anti-autoimmune activities, and stimulation of antioxidant pathways and provided that daily safe intake level is not exceed, its use in higher doses could be therapeutic in ASD. Targeted blood level is $50 \mathrm{ng}-80 \mathrm{ng} / \mathrm{ml}$ to treat vitamin D deficiency in children with ASD [1]. 


\section{FOLIC ACID}

\subsection{Folate Metabolism}

Folates include a family of compounds chemically related to folic acid. All tissue folates are in the polyglutamate form. Metabolizing the polyglutamate forms of the folates is necessary for their biological activities. Polyglutamate forms are far more effective substrates for dependent enzymes than monoglutamate forms. It is the active coenzyme structure of the tetrahydrofolate vitamin [24]. Folate is necessary for normal metabolic processes as the methyl group donor. It also plays an important role in methionine syntheses. A sufficient amount of folate prevents excessive accumulation of serum homosistein levels [25].

Folate has many roles as a coenzyme. Its function is to transfer and accept carbon units. The functions of folate in mammals are generally to retrieve mono carbon units from serine and transfer it to purines and pyrimidine biosynthesis. Therefore, folate enzymes are essential for DNA synthesis. Folate coenzymes are necessary for the synthesis of methionine and other cellular compounds [5]. Different genetic polymorphisms occurring in folate pathway key enzymes are related to autism. These abnormalities cause deterioration in the production of folate cycle metabolites, a decrease in 5methyltetrahydrofolate production, and transmission of folate across the blood-brain barrier [26]. As a result, folic acid is necessary for $\mathrm{CpG}$ methylation and gene expression. Low folate levels reduce genomic methylation and negatively affect gene expression during developmental processes such as cell growth [7]. Genetic polymorphisms in methylenetetrahydrofolate reductase and dihydrofolate reductase as well as a reduction in folate transmission are related to autism [26].

\subsection{The role of folate in autism occurrence}

Folic acid supplementation may be related to some abnormalities in children. Lymphoblastoid cells exposed to folic acid supplementation may cause changes in gene expression [27]. In addition, epigenetic changes during gestational development may affect DNA methylation levels in the fetus resulting in various neuropsychiatric situations affecting brain development due to the changes in gene expression [28]. There are significantly different methylated areas in the cerebral hemispheres of young mice that had higher maternal folic acid exposure than the ones with lower maternal folic acid exposure [29]. Abnormalities in the frontal lobe may affect brain development and ASD [30].

Dietary folic acid supplementation during pregnancy is a successful community health program instituted to prevent neural tube defects. However, encouraging folic acid intake with supplementation before and during pregnancy and enriching certain grains with folic acid highly increases folic acid levels [25]. Some epidemiological studies show that folic acid supplementation protects against ASD, whereas others report that it increases ASD risk and prevents neurocognitive development in children whose mothers used synthetic folic acid. One study determined the effect of high folic acid intake on cerebral hemisphere genes in rats. One week before mating and during pregnancy, female rats were given $20 \mathrm{mg} / \mathrm{kg}$ (control group) or $20 \mathrm{mg} / \mathrm{kg}$ folic acid. On the first postnatal day, cerebral hemisphere tissues were obtained and assessed. Pups whose mothers received high levels of folic acid had sexspecific changes in gene expression [31].Another study found that gene expression in the cerebral hemisphere changed in mouse pups exposed to high levels of folic acid during pregnancy and behavioral disorders such as anxiety and hyperactivity were observed [32].

A comparison of the autism incidence rate published in a study conducted between 1976 and 1997, the prescription rate of vitamins containing $1 \mathrm{mg}$ folic acid, and the prescription rate of any pediatric vitamin containing folic acid was assessed. A significant correlation between autism incidence 
and prenatal vitamin use and a weak correlation with pediatric vitamin use was found. The same study showed that very little or high consumption of folic acid may damage nerve tissue [33].

Human dihydrofolate reductase (DHFR) expression is relatively low and has a variable nature in liver. The highest reliable level of folic acid which is tolerable is $1000 \mathrm{mcg} / \mathrm{day}$. High intake of folic acid may result in non-metabolized folic acid in the blood. A dose-related effect occurs when the intake level exceeds $200 \mathrm{mg} / \mathrm{day}$. More than $1 \mathrm{mg}$ folic acid intake results in non-metabolized folic acid, although there were breaks between dosages. Non-metabolized high serum folic acid levels have harmful effects on dihydrofolate enzyme expression, folic acid intake regulation in kidney and intestinal epithelium, neural tissues, and mental health [25].

Neural development is sensitive to maternal metabolic conditions. A high amount of folic acid supplementation in a pregnant woman's diet causes changes in brain synaptic transmission and increased crisis risks [34]. In pups born from mice with high folic acid intake, gene expression in the cerebrum changed and these pups showed cognitive changes such as anxiety and hyperactivity [32]. Another study found that high folic acid nutrition during pregnancy caused altered regulation in gene expression of ASD-related and neurodevelopmental genes [35]. Mean levels of folic acid are reliable markers of health problems, however high levels may alter the epigenetic network [25].

Folate and biopterin pathway members interact. Product levels from both pathways and cerebrospinal fluid biopterin levels may change, which can be treated with tetrahydrobiopterin, a critical component in serotonin, dopamine, and neurotransmitter synthesis [7]. Folate and folic acid are essential for basic cellar processes including DNA replication and protein methylation. Therefore, folic acid intake has an impact dependent on time and dosage. There are different hypotheses to explain the relationship between folic acid and autism. One hypothesis is that serum folate receptor alpha (FR $\alpha)$ autoantibodies (FRAs) block the folate binding area of the membrane-linked folate receptors on choroid epithelial cells [5]. FRAs are related to developmental diseases including fetal abnormalities and cerebral folate deficiency [36]. Another study assessed the effects of FR $\alpha$ antibody exposure before and after weaning on subsequent behaviors during pregnancy in rats. The study found that the anxiety level of the rats exposed to FR $\alpha$ antibodies before weaning were high, whereas the rats exposed to FR $\alpha$ antibodies in later stages did not show behavioral disorders [37].

Metabolic abnormalities regarding folate metabolism are seen in most ASD studies [25]. Cerebral folate deficiency (CFD) syndrome is a neurometabolic disorder characterized by low 5methyltetrahydrofolate (5MTHF) levels in cerebrospinal fluid, despite normal levels of systematic folate. CFD distorts the transmission of folate across the blood-brain barrier. Although the biological mechanism between ASD and CFD is not fully known, it can be explained by some symptoms observed in autism such as folate deficiency in the central nervous system, DNA methylation in the brain, and oxidative stress. Some children with CFD showed relief in autism symptoms with folinic acid treatment [38].

One of the most important abnormalities regarding ASD is the presence of autoantibodies against FR $\alpha$. Energy reliant receptors are used for the transportation of folate to the blood-brain barrier. Autoantibodies can bind to these receptors and inhibit their function [26]. A control group was consisted of 75 patients with autism and 30 patients without autism. The blocked FR $\alpha$ auto antibody percentage was $47 \%$ in the patients with autism and 3\% in the control group [39]. Another study showed that high dosage of folinic acid treatment significantly ameliorated the linguistic area of children with ASD such as oral communication, receiver and enunciative fields. According to a study, FRAs were found in 
75.3\% of 93 children with autism whose serum folate antibodies were measured. Interestingly, an amelioration in oral communication, receptive and expressive language, attention, and repetitive behaviors of children with FRAs treated with oral leucovorin (folinic acid) calcium for approximately 4 months was observed. The authors recommended all children with autism have an FRA test [38]. Similarly, communication, learning, and cognitive disorders occurred in rats exposed to FRAs during pregnancy, which could be prevented by folinic acid treatment [36]. Another study found that rat pups exposed to FR $\alpha$ antibodies during pregnancy and lactation showed intensive behavioral problems [37]. Genetic polymorphisms are often observed in the enzymes necessary to metabolize folate in autism. The MTHF reductase (MTHFR) enzyme is used in folic acid methylation to turn it into a usable form. The survival rate of children with the MTHFR C677T polymorphism can be increased by boosting maternal folic acid intake. These children have reduced enzyme activity and increased neurological recession and autism without folic acid supplementation. Therefore, mutations in the MTHFR enzyme are related to autism. According to proposed hypotheses, the increase in folic acid supplementation boosts the birth rate of children who had mutations in the MTHFR enzyme. Normal or reduced levels of folic acid in a fetus with reduced level of MTHFR enzyme increase the miscarriage risk. Thus, it is necessary to provide children with folic acid to compensate for the reduced enzyme activity after birth. If folic acid supplementation for the children whose MTHFR enzymes are mutated does not occur, it poses a risk for autism development [40].

Excessive folic acid can change behavioral characteristics during cerebral development by changing gene expression. C677T-MTHFR interacts with dietary folate to change the balance between DNA and methionine biosynthesis. Due to insufficiencies in the diet, reductions in genomic methylation capacity can negatively affect gene expression. Low folate levels can affect methionine and S-adenosylmethionine and change methyl group synthesis [7].

\section{Conclusions}

Sufficient levels of folate before and at the early stages of pregnancy has a general protective effect. However, high levels can cause undesired effects on DNA methylation during periods of rapid cell division, such as prenatal development. Non-metabolized folic acid can directly change the behaviors of the neurons in certain neural cycles during development. High folic acid supplementation changes genomic functions. One must be careful because folic acid supplementation with folic acid enriched grains can have harmful effects causing excessive folic acid accumulation.

Low levels of vitamin D during pregnancy, postnatal period and early childhood are considered to be a risk factor for ASD which is one of neurodevelopmental disorders. Exposure to vitamin D deficiency in early period may contribute etiology of ASD by interacting with other factors. Vitamin D may directly influence main symptoms of ASD. Therefore, vitamin D supplementation may be beneficial for reducing symptoms seen in patients with ASD. It should be a part of treatment procedure for ASD to routinely assess serum vitamin D level and to supplement sufficient vitamin D. However, conducting long-term controlled clinical studies with large sample size will help to understand potential role of vitamin D and folic acid. 


\section{References}

[1]Coşkun, S., Şimşek, Ş., Camkurt, M.A., Çim, A., Çelik, S.B., “Association of Polymorphisms in the Vitamin D Receptor Gene and Serum 25-hydroxyvitamin D Levels in Children with Autism Spectrum Disorder", Gene, 588,109-114,2016.

[2]Stubbs, G., Henley, K., Green, J., “'Autism: will vitamin d supplementation during pregnancy and eearly childhood reduce the recurrence rate of autism in newborn siblings?" Medical Hypotheses, 88,74-78,2016.

[3]Cannell, J.J., “Vitamin D and Autism, What's New? ”, Rev Endocr Metab Disord, 2017 doi:10.1007/s11154-017-9409-0.

[4] Castro, K., Klein, L.S., Baronio, D., Gottfried, C., Riesgo, R., Perry, I.S., “'Folic acid and autism: What do we know?"' Nutritional Neuroscience, 19(7), 310-317, 2016.

[5]Neggers, Y., ' 'The relationship between folic acid and risk of autism spectrum disorders", Healthcare, 2, 429-444,2014.

[6]Kałużna-Czaplińska, J., Socha, E., Rynkowski, J.B., "Vitamin supplementation reduces excretion of urinary dicarboxylic acids in autistic children', Nutrition Research, 31,497-502, 2011.

[7]Lucock, M., Leeming, R., “'Autism, seasonality and the environmental perturbation of epigenome related vitamin levels", Medical Hypotheses, 80,750-775,2013.

[8]Hashemzadeh, M., Moharreri, F., Soltanifar, A., ' Comparative study of vitamin d levels in children with autism spectrum disorderand normal children: a case-control study", Fundamentals of Mental Health, 17(4),197-201,2015.

[9]Jia, F., Wang, B., Shan, L., Xu, Z., Staal, W.G., Du, L., “Core symptoms of autism improved after vitamin D supplementation", Pediatrics, 135( 1),196-198,2015.

[10]Cannell, J.J., Grant, W.B., "What is The Role of Vitamin D in Autism? ', Dermato-Endocrinology, 5(1), 199-204,2013.

[11]Mazahery, H., Camargo, C.A., Conlon, C., Beck, K.L., Kruger, M.C., Hurst, P.R., "'Vitamin D and autism spectrum disorder: a literature review", Nutrients, 8,236,2016, doi:10.3390/nu8040236.

[12]Koc`ovska, E., Elisabeth, F., Billstedt, E., Minnis, H., Gillberg, C., "Vitamin D and autism : Clinical Review", Research in Developmental Disabilities,33,1541-1550,2012.

[13]Feng, J., Shan, L., Du, L., Wang, B., Li, H., Wang, W., et al., "Clinical improvement following vitamin $\mathrm{D}_{3}$ supplementation in autism spectrum disorder", Nutritional Neuroscience, 2016, DOI 10.1080/1028415X.2015.1123847.

[14]Zerbo, O., Losif, A.M., Delwiche, L., Walker, C., Hertz-Picciotto, I., “'Month of conception and risk of autism', Epidemiology, 22,469-475, 2011.

[15]Whitehous, A.J., Holt, B.J., Serralha, M., Holt, P.G., Kusel, M.M., Hart, P.H., 'Maternal serum vitamin D levels during pregnancy and off spring neurocognitive development", Pediatrics, 129,485493, 2012.

[16]Morales, E., Guxens, M., Llop, S., Rodríguez-Bernal, C.L., Tardón, A., Riaño, I., et al., “'Circulating 25-hydroxyvitamin $\mathrm{D}_{3}$ in pregnancy and infant neuropsychological development", Pediatrics, 130,913-920,2012.

[17]Eylesa, D., Burnea, T., McGrath, J., "Vitamin D in fetal brain development", Seminars in Cell \& Developmental Biology, 22,629- 636, 2011. 
[18]Mostafa, G.A., Al Shehab, A., Fouad, N.R., “ Frequency of CD4+CD25 high regulatory t cells in the peripheral blood of egyptian children with autism", J Child Neurol, 25,328-35,2010.

[19]Holló, A., Clemens, Z., Kamondi, A., Lakatos, P., Szücs, A., “Correction of vitamin D deficiency improves seizure control in epilepsy: a pilot study", Epilepsy Behav, 24,131-133,2012.

[20]Patrick, R.P., Ames, B.N., "Vitamin D hormone regulates serotonin synthesis. Part 1: relevance for autism', FASEB J, 28,1-16,2014.

[21]Humble, M.B., Gustafsson, S., Bejerot, S., “'Low serum levels of 25-hydroxyvitamin D (25-OHD) among psychiatric out-patients in sweden: relations with season, age, ethnic origin and psychiatric diagnosis', J. Steroid Biochem. Mol. Biol, 121,467-470,2010.

[22]Saad, K., Abdel-Rahman, A.A., Elserogy, Y.M., Al-Atram, A.A., Cannell, J.J., Bjorklund, G., et al., "Vitamin D status in autism spectrum disorders and the efficacy of vitamin D supplementation in autistic children', Nutr. Neurosci, 346-351, 2015.

[23]Guler, S., Yesil, G., Ozdil, M., Ekici, B., Onal, H., “'Sleep disturbances and serum vitamin D levels in children with autism spectrum disorder', Int J Clin Exp Med, 9(7),14691-14697, 2016.

[24]Shane, B., "Folate and vitamin $\mathrm{B}_{12}$ metabolism: Overview and interaction with riboflavin, vitamin $\mathrm{B}_{6}$, and polymorphisms", Food and Nutrition Bulletin, 29(2), 5-16, 2008.

[25]Wiens, D., DeSoto, M.C., 'Is high folic acid intake a risk factor for autism?-A Review', BrainSci, 7 , 149, 2017, doi:10.3390/brainsci7110149

[26]Frye, R.E., Rossignol, D.A., “'Treatments for biomedical abnormalities associated with autism spectrum disorder', Front Pediatr, 27(2),66,2014, doi: 10.3389/fped.2014.00066.

[27]Junaid, M.A., Kuizon, S., Cardona, J., Azher, T., Murakami, N., Pullarkat, R.K., Brown, T.W., "Folic acid supplementation dysregulates gene expression in lymphoblastoid cells-Implications in nutrition', Biochem. Biophys. Res. Commun, 412,688-692, 2011.

[28]Balion, C., Kapur, B.M., "Folate:clinical utility of serum and red blood cell analysis', Clin. Lab. News, 37, 8-10, 2011.

[29]Baura, S., Kuizon, S., Chadman, K.K., Flory, M.J., Brown, W.T., Junaid, M.A., “'Single base resolution of mouse offspring brain methylome reveals epigenome modifications caused by gestational folic acid', Epigenetics Chromatin, 7,1-15, 2014.

[30]Wong, C.C., Meaburn, E.L., Ronald, A., Price, T.S., Jeffries, A.R., Scalkwyk, L.C., Plomin, R., Mills, J., "Methyomic analysis of monozygotic twins discordant for autism spectrum disorder and related behavioral traits"', Mol. Psychiatry, 19,495-503, 2014.

[31]Barua, S., Kuizon, S., Brown, W.T., Junaid, M.A., "High gestational folic acid supplementation alters expression of imprinted and candidate autism susceptibility genes in a sex-specific manner in mouse offspring', J Mol Neurosci, 58,277-286, 2016.

[32]Barua, S., Chadman, K.K., Kuizon, S., Buenaventura, D., Stapley, N.W., et al. “Increasing maternal or post-weaning folic acid alters gene expression and moderately changes behavior in the offspring', PLoS ONE, 9(7), 2014, e101674. doi:10.1371/journal.pone.0101674

[33]Beard, C.M, Panser, L.A., Katusic, S.K., 'Is excess folic acid supplementation a risk factor for autism? ', Medical Hypotheses, 77,15-17, 2011.

[34]Girotto, F., Scott, L., Avchalumov, Y., Harris, J., Iannattone, S., Drummond-Main, C., Tobias, R., Bello-Espinosa, L., Rho, J.M., Davidsen, J., et al., "High dose folic acid supplementation of rats alters synaptic transmission and seizure susceptibility in offspring", Sci. Rep, 3,1465, 2013, doi:

10.1038/srep01465 
[35]Barua, S., Kuizon, S.K., Chadman, K., Brown, W.T., Juniad, M., “Microarray analysis reveals higher gestational folic acid alters expression of genes in the cerebellum of mice offspring-A pilot study', Brain Sci, 5,14-31, 2015.

[36]Desai, A., Sequeira, J.M., Quadros, E.V., ' 'Prevention of behavioral deficits in rats exposed to folate receptor antibodies: implication in autism”, Molecular Psychiatry, 22,1291-1297, 2017.

[37]Sequeira, J.M., Desai, A., Berrocal-Zaragoza, M.I., Murphy, M.M., Fernandez-Ballart, J.D., Quadros, E.V., "Exposure to folate receptor alpha antibodies during gestation and weaning leads to severe behavioral deficits in rats: a pilot study", PLOS ONE, 11(3), 2016, e0152249. doi:10.1371/journal.pone.015224

[38]Frye, R.E., Sequeira, J.M., Quadros, E.V., James, S.J., Rossignol, D.A., “'Cerebral folate receptor autoantibodies in autism spectrum disorder', Molecular Psychiatry, 18,369-381, 2013.

[39]Ramaekers, V.T., Quadros, E.V., Sequeira, J.M., "Role of folate receptor autoantibodies in infantile autism”, Mol Psychiatry, 18,270-1, 2013,doi:10.1038/mp.2012.22

[40]Currenti, S.A., "Understanding and determining the etiology of autism', Cell Mol Neurobiol, 30,161$171,2010$. 